\title{
EDITORIAL
}

\section{Functional foods}

\author{
European Journal of Clinical Nutrition (2010) 64, 657-659; \\ doi:10.1038/ejcn.2010.101
}

Humankind has always been interested in food. This, although a platitude, is worth remembering. The science of functional foods is the convergence of two major events in our lives-diet and health. The association between food and disease is widely recognized as the bedrock of preventive nutrition. The concept of 'functional foods' is often cited as a newly emerging field. However, this idea was first described in the ancient Vedic texts from India, and in Chinese traditional medicine. The vision to develop functional foods reflects the oriental philosophy that: 'Medicine and food have a common origin'.

The conviction to develop functional foods first emerged in Japan in the 1980s when faced with escalating health-care costs. The Ministry of Health and Welfare initiated a regulatory system to approve certain foods with documented health benefits (Arai, 1996). Its primary objective was to improve the health of the nation's ageing population. In 1984, the Ministry of Education, Science and Culture, an ad hoc group in Japan commenced a national project to explore the link between food and medical sciences (Ohama et al., 2006). The term 'functional food' first appeared in 1993 in the Nature news magazine under the heading 'Japan explores the boundary between food and medicine' (Swinbanks and O'Brien, 1993).

Functional food is essentially a marketing term and globally, it is not recognized by law. Several definitions for functional foods exist. These include, that given by Health Canada: 'Similar in appearance to conventional food, consumed as part of the usual diet, with demonstrated physiological benefits, and/or to reduce the risk of chronic disease beyond basic nutritional functions' (Health Canada, 2000). Other definitions include that from the International Food Information Council (IFIC), that is functional foods are, 'foods or dietary components that may provide a health benefit beyond basic nutrition' (Bagchi, 2008). The International Life Sciences Institute of North America (ILSI) has defined functional foods as, 'foods that by virtue of physiologically active food components provide health benefits beyond basic nutrition' (Bagchi, 2008). The European Commission Concerted Action on Functional Food Science in Europe regards a food as functional, 'if it is satisfactorily demonstrated to affect beneficially one or more target functions in the body, beyond adequate nutritional effects, in a way that is relevant to either an improved state of health and well-being and/or reduction of risk of disease' (Consensus document, 1999).

Today, Japan is the only country that recognizes functional foods as a distinct category, and the Japanese functional food market is now one of the most advanced in the world. Known as foods for specified health use (FOSHU), these are foods composed of functional ingredients that affect the structure and/or function of the body and are used to maintain or regulate specific health conditions, such as gastrointestinal health, blood pressure and blood cholesterol levels (Hosoya, 1998). As of July 2008, nearly 500 food products had been granted FOSHU status in Japan.

In 1912, Casimir Funk presented a seminal paper proposing the 'Vitamine' theory (McCollum, 1957). He proposed that the absence of certain minute substances in foods rather than the presence of germs caused disease. The theory and concept that he developed has had a direct bearing on the development of functional foods. The concept of functional foods has now been extended to include food constituents that reduce the risk of chronic disease (Plat and Mensink, 2001). Today we are at a new frontier in nutritional science. The transition from 'adequate' to 'optimal' nutrition. It is here that functional foods will have a pivotal role in reducing diet-related chronic diseases.

Functional foods may be broadly grouped into the following:

- Conventional food containing naturally occurring bioactive substance. An example could be $\beta$-glucan in oat bran to lower blood cholesterol;

- Foods that have been modified, by enrichment or other means, with bioactive substances. An example could be margarine that contains added phytosterol that is known to lower serum cholesterol;

- Synthesized food ingredients, such as some specialized carbohydrates intended to have probiotic effects.

A functional food can be (1) a natural food, (2) a food to which a component has been added, (3) a food from which a component has been removed, (4) a food where one or more components has been modified, (5) a food in which the bioavailability has been modified or (6) any combination of these. Examples of these are shown in Table 1.

A food product can be made functional by using any of the five approaches listed below:

(1) Eliminating a component known to cause or identified as causing a deleterious effect when consumed (for example, an allergenic protein). 
Table 1 Categories of functional foods

\begin{tabular}{ll}
\hline Category & Example \\
\hline Basic food & Carrots (containing the anti-oxidant $\beta$-carotene) \\
Processed foods & Oat bran cereal \\
Processed foods with added ingredients & Calcium-enriched fruit juice \\
Food enhanced to have more of a functional component & Tomatoes with a higher levels of lycopene \\
Isolated, purified preparations of active food ingredients (dosage form) & Isoflavones from soy \\
& $\beta$-Glucan from oat bran \\
\hline
\end{tabular}

Adapted from Arvanitoyannis and Van Houwelingen-Koukaliaroglou (2005).

(2) Increasing the concentration of a component naturally present in food to a point at which it will induce predicted effects (for example, fortification with a micronutrient to reach a daily intake higher than the recommended daily intake).

(3) Adding a component that is not normally present in most foods and is not necessarily a macronutrient or a micronutrient, but for which beneficial effects have been shown (for example, non-vitamin antioxidant or prebiotic fructans).

(4) Replacing a component, usually a macronutrient (for example, fats), intake of which is usually excessive and replacing it with a component for which beneficial effects have been shown (for example, modified starch).

(5) Increasing bioavailability or stability of a component known to produce a functional effect or to reduce the disease-risk potential of the food.

One of the examples often quoted within the functional food sector is the introduction of margarine spreads fortified with plant sterols in the UK. Although there is limited information related to the impact of diet-based intervention on disease prevention and health-care cost reduction, a few case studies are available to support the use of functional foods to improve the health of the populations. A reduction of low-density lipoprotein cholesterol by $10-15 \%$ was observed through the consumption of plant stanol esters at levels of 2-3 g per day (Nguyun, 1999). If this risk reduction is achieved in practice, heart disease patient numbers in the UK would reduce by 2500000 and save the UK health-care system $£ 433$ million. The National Health Service in the UK estimated that these products have the potential to lower the health-care costs for cardiovascular disease by $£ 100$ million per year.

Despite the emerging interest and global consumption of functional foods, the recent opinion of European Food Safety Authority (EFSA) on article 13.1 for health claims has caused considerable concern and consternation among nutritionists and food manufacturers alike (Hughes, 2009). Of the 416 claims evaluated, fewer than $2 \%$ were approved, a rejection rate that calls into question the process itself. Claims related to antioxidants, bowel function, neurological function and glycaemic index were rejected. The rejection of carbohydrates and their glycaemic indices appears to be at variance with other international bodies. The World Health Organization, for example, not only recognizes the concept of glycaemic index, it advocates its wide usage (FAO/WHO, 1998). Yet EFSA states that 'carbohydrates that induce a low/reduced glycaemic response and carbohydrates with a low glycaemic index $(<55)$, which are the subject of the health claims are not sufficiently characterised'. This is hard to reconcile as the carbohydrate content of several foods has not only been carefully characterized but also recognized as being metabolized and absorbed differently. Even more curious is the approval of $\beta$-glucan for lowering cholesterol but not for lowering blood glucose. These anomalies highlight the challenges ahead. EFSA urgently needs to balance scientific judgement and consumer protection with the promotion and nurturing of food innovation within Europe. There is an urgent need for EFSA and the food industry to recalibrate each other's expectations. If not, EFSA will be perceived as a harbinger of doom for the industry and scientists alike, stifling creativity and ultimately the consumer will be the loser.

As the head of the Functional Food Centre in Oxford whose primary goal is to evaluate and assess functional benefits of foods and ingredients and to provide evidencebased science to substantiate health claims, I welcome the importance given to functional foods by the European Journal of Clinical Nutrition by publishing this special issue on functional foods based on peer-reviewed reviews and articles accepted by the Journal. EJCN should be commended as it has continued to support this growing new area of nutritional science by consistently promoting the publication of evidence-based studies in this important field.

\section{CJ Henry \\ Human Nutrition, Functional Food Centre, Oxford Brookes University, Oxford, UK E-mail: jhenry@brookes.ac.uk}

\section{References}

Arai S (1996). Studies on functional foods in Japan. Bioscience Biotechnol Biochem 60, 9-15.

Arvanitoyannis IS, Van Houwelingen-Koukaliaroglou M (2005). Functional foods: a survey of health claims, pros and cons, and current legislation. Crit Rev Food Sci Nutr 45, 385-404. 
Bagchi D (ed). (2008). Neutraceutical and Functional Food Regulations. Elsevier: New York.

Consensus Document (1999). Scientific concepts of functional foods in Europe consensus document. $\mathrm{Br} J$ Nutr 81, S1-S27.

Food and Agriculture Organisation/World Health Organisation (1998). Carbohydrates in human nutrition: report of a joint FAO/WHO expert consultation, FAO Food and Nutrition paper 66, Rome.

Health Canada (2000). Standards of evidence for evaluating foods with health claims. Fact sheet 1 . November 2000.

Hosoya N (1998). Health claims in Japan-foods for specified health uses and functional foods. J Nutr Food 1, 1-11.
Hughes N (2009). Health claims thrown into disarray by EFSA rejections. The Grocer, 10 October: 12-13.

McCollum EV (1957). A History of Nutrition. The Riverside Press: Cambridge, MA.

Swinbanks D, O'Brien J (1993). Japan explores the boundary between food and medicine. Nature 364, 180.

Nguyun TT (1999). The cholesterol-lowering action of plant stanol esters. J Nutr 129, 2109-2112.

Ohama H, Ikeda H, Moriyama H (2006). Health foods and foods with health claims. Toxicology 221, 95-111.

Plat J, Mensink RP (2001). Effects of plant sterols ands stanols on lipid metabolism and cardiovascular risk. Nutr Metab Cardiovasc Dis 11, 31-40. 